\title{
Controllability pre-verification of silicone soft robots based on finite-element method
}

\author{
G. Zheng, O. Goury, M. Thieffry, A. Kruszewski, C. Duriez
}

\begin{abstract}
Soft robot is an emergent research field which has variant promising applications. However, the design of soft robots nowadays still follows the trial-and-error process, which is not at all efficient. This paper proposes to design soft robots by pre-checking controllability during the numerical design phase. Finite-element method is used to model the dynamics of silicone soft robots, based on which the differential geometric method is applied to analyze the controllability of the points of interest. Such a verification is also investigated via model order reduction technique and Galerkin projection. The proposed methodology is finally validated by numerically designing a controllable parallel soft robot.
\end{abstract}

\section{INTRODUCTION}

Soft robots are rightly able to adjust their shapes to suit the task and their environments [1]. The term "soft" means the robots' mechanical function relies on using deformable structures in a way similar to the biological world and organic materials. The use of deformable materials makes soft robots very compliant, which provides positive outcomes that are complementary to traditional rigid robotics. Due to their compliance, they can access to fragile parts of an environment by applying minimal pressure. Their large number of degrees of freedom and actuators ease the maneuvering through soft and confined spaces. These make soft robots relevant for medical and surgical robotics [2], manipulation of fragile objects, domestic robotics with safer interactions with humans, arts and entertainment.

Up to now, the design of soft robot is based on nature, including the elephant's trunk, the octopus, and the worm [3]. Compared to rigid robots for which several softwares and simulation tools, such as Gazebo [4], have been developed to facilitate its numerical design procedure, the design of soft robots nowadays still follows the trial-and-error process, which is not at all efficient, or even time-consuming, since the functional verification stage can be only effectuated after the soft robots have been made and assembled. Also, it is expensive/wasteful in the sense that many materials cannot be reused if the functionality of fabricated soft robots is not satisfactory. Therefore, the requirement of an efficient numerical methodology for soft robot design is necessary.

As a goal, the final prototype of soft robot should be controllable. Therefore, this issue deserves to be taken into account during the numerical design phase. The main contribution of this paper is to investigate such a methodology to

This work is supported in part by project Inventor (I-SITE ULNE, le programme d'Investissements d'Avenir, Métropole Européenne de Lille), and by project VALID (CPER DATA). G. Zheng, O. Goury, M. Thieffry, A. Kruszewski, C. Duriez are with Defrost team, INRIA LilleNord Europe, 40, avenue Halley, 59650 Villeneuve d'Ascq, France. firstname.namedinria.fr numerically design controllable soft robots. In this paper, we limit our investigations on soft robot made by solid deformable materials, such as silicone. Therefore finiteelement method (FEM) is used in the proposed methodology to model the desired soft robots. Such a choice has been validated by several designs of soft robots [5], [6], [7], [8], [9]. After that, using differential geometric method [10], [11], sufficient condition is deduced to judge whether the selected points of interest of soft robot can be controllable or not. Such a condition is discussed both for high-dimensional FEM model and also for reduced-order model, which is obtained by applying model order reduction (MOR) technique. In the literature, MOR is a topic which has been studied in very different domains, including control community [12], signal processing [13], mechanics [14] and so on. Different techniques are proposed, such as reduced basis methods [15], proper orthogonal decomposition [16], balanced truncation [17], Krylov subspace methods [18], proper generalized decomposition [19] etc. Roughly speaking, MOR enables us to approximate the high-dimensional system with a lower one by preserving satisfactory properties. In the proposed methodology, proper orthogonal decomposition will be used to deduce the reduced-order model via Galerkin projection [20]. An iterative procedure is proposed in this paper to numerically design the soft robot by pre-checking the controllability of the selected points of interest. The proposed methodology is finally applied to numerically design a parallel soft robot for the purpose of highlighting its effectiveness.

\section{Modeling AND PROBlem StATEMEnT}

Before stating the problem of soft robot design, let us firstly take a look at the procedure when designing rigid robots. Imaging that we are going to design a rigid manipulator, as shown in Figure 1 (Left), we can then follow the following conventional procedure:

[Step 1] Draw the desired mechanics, validate the kinematics and design the mechatronic components;

[Step 2] Deduce automatically the corresponding dynamical model (i.e., ordinary differential equation (ODE), since it is assumed to be rigid);

[Step 3] Analyze the properties based on the obtained model to judge whether such a configuration can satisfy user's requirements, for example, capability to be controlled;

[Step 4] If yes, we can then synthesize and test the controller for the numerical model (via numerical simulation in a virtual environment);

If no, go back to Step 1 to modify the configuration 
and repeat the same procedure till the design is satisfactory.

This is an intuitive and efficient way to design any form of rigid robots. A natural and interesting question is: 'Can we have the similar efficient procedure to design soft robots?'. It is a huge challenge to answer this question, since the flexible characteristic of soft robots makes the modeling (Step 2) quite difficult, and this prevents the developments of Step 34. The objective of this paper is to investigate the difficulties of this challenge and to propose a first feasible solution, even if it is limited to certain types of soft robots.
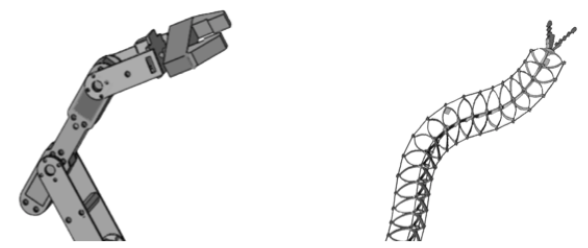

Fig. 1. Robot design scenarios. Left: Rigid manipulator, actuated by motors; Right: Soft manipulator, actuated by cables

To design a general form of soft robots with solid deformable materials (such as silicone), for example a deformable manipulator as shown in Figure 1 (Right), how can we model such an irregular design mathematically? Since the robot is flexible, it is natural to think about the modeling by partial differential equation (PDE). Such a choice however suffers from the following problems

1) It is feasible only for simple cases (such as linear domain, homogeneous parameters), and it fails to deduce automatically the PDE model from irregular shape (for example the soft manipulator in Figure 1 or the parallel soft robot presented in Figure 3);

2) Generally, the dynamics of soft robots is nonlinear, and this nonlinearity makes the modeling by nonlinear PDE more complex, for which the property analysis becomes quite difficult;

3) Also, the actuators equipped in soft robots might provide either boundary or domain (distributed) control (such as tendon at a point or penetrates inside the robot body, or pressure actuator...), and this will complicate again the upcoming analysis of properties for nonlinear PDE;

4) Another weaknesses of the modeling via nonlinear PDE would be the lack of well-developed results/software which enable us to efficiently pre-check the properties of the desired soft robots by numerical methods. Note that this pre-check needs to be repeated each time when the design has been modified (similar to Step 4 for rigid robot design).

In fact, these problems, related to the modeling of deformation for solid continuum materials, have already been identified in mechanics and addressed using numerical methods, such as FEM. FEM allows to obtain the dynamics, even for irregular shapes and complex boundary/domain conditions. So it can capture different types of actuators including boundary and domain control. In this sense, FEM is a good candidate to model soft robots made by solid deformable materials. Also, there already exist certain softwares which enable us to automatically generate the FEM model for any shape, and efficiently update the FEM model when modification is made.

For those reasons, we limit our study on a special class of soft robots.

\section{Assumption 1. It is assumed that}

1) the soft robot is made by homogeneous solid deformable materials with known homogeneous properties (such as elasticity, constitutive law and so on);

2) FEM can provide precisely approximate model of soft robots, by choosing small size of mesh;

3) The robot to be designed is limited by its workspace and the outputs of actuators are physically bounded.

The above assumption imposed certain limitations of our study. However, we would like to remark the following facts.

- The item 1) of Assumption 1 might be satisfied, since the users normally know in advance which material (and its properties) will be used to fabricate soft robot. However, the requirement of homogeneous property and material seems restrictive for the fabrication phase. In fact, non-homogeneous properties can be also possible once the user knows well its property and can integrate it into FEM. The item 1) is imposed only for the simplicity reason.

- Secondly, with the known properties of solid deformable materials, it is possible to obtain a precise approximate model via FEM if the mesh is sufficient small. In other words, the item 2) of Assumption 1 is also feasible. Of course, smaller the mesh is chosen, greater the dimension of FEM model will be. And this will heavily increase the computation time. However, as we will see in Section IV, MOR can be applied to highly reduced the dimension of FEM model;

- Generally, the item 3) of Assumption 1 is always satisfied for any mechanical system, including soft robots.

Besides the flexible materials, actuator (to drive soft robots) is another important issue to be investigated. The type of actuators and where they are mounted will determine the controllability (possibility of regulation) of the designed soft robots. In this sense, it is necessary to simulate numerically the soft robots, for which a pre-analysis is crucial to determine the type and the place of the integrated actuators by checking the controllability. These properties are important when considering controller design problem in the upcoming stage.

When designing a soft robot, since the number of actuators is limited while the number of element is huge, logically it is not possible to control all elements at the same time. In most of cases, we only want to control certain points of interest of soft robots (for example the end-effector of the flexible manipulator). Therefore, in the design phase, we are looking for a feasible configuration: with minimum 
number of actuators to achieve the easy control of certain points of interest. Consequently, this paper tries to answer the following problem: Given a configuration of soft robot with the equipped actuators (in a virtual environment), satisfying Assumption 1, does such a configuration enable us to control certain points of interest?

\section{ANALYSis BASED ON FinItE-ELEMENT MODEL}

\section{A. Modeling of soft robots via FEM}

Under Assumption 1, for a given configuration of soft robot, with the equipped actuators, we can then discretize its space by using finite number of fine elements to deduce its dynamical model. Following the second law of Newton, we can use the following nonlinear model to describe its behavior [5]:

$$
M(q) \ddot{q}+D(q, \dot{q}) \dot{q}+K(q) q=H^{T}(q) \lambda
$$

where $q \in \mathbb{R}^{n_{q}}$ is the position of the nodes of the mesh, $M(q)$ is mass matrix which is always invertible, $D$ is damping matrix, and $K(q)$ represents stiffness matrix.

The damping matrix $D(q, \dot{q})$ and the tangent stiffness matrix $K(q)$ are arose from the internal forces of the soft robot, which depends on the constitutive law of the material the soft robot is made of. The damping matrix is often taken as being a linear combination of the mass and stiffness matrices: $D=\alpha M+\beta K$, with the coefficients $\alpha$ and $\beta$ being the Rayleigh damping coefficients [7]. $H(q)$ represents the force directions (including actuators from the robot itself), and is usually sparse, as it has only non-zero values at the points where the actuators are applied. $\lambda$ represents the magnitude of the actuators.

\section{B. System transformation and differential geometry}

Under Assumption 1, given any configuration of soft robot with the equipped actuators, we can derive the dynamical model (1) by applying FEM. The objective is then to check the controllability of certain chosen points, which in fact is a well-known notion in control community. In order to be coherent with symbols used in control community, let us note

$$
x=\left[\begin{array}{c}
x_{1} \\
x_{2}
\end{array}\right]=\left[\begin{array}{c}
q \\
\dot{q}
\end{array}\right], u_{i}=\lambda_{i}
$$

and denote by $y \in \mathbb{R}^{p}$ the chosen points of interest:

$$
y=h(x)
$$

It is worthy noting that normally $y$ is a linear function of $x$ since the points of interest can be freely chosen by the designer. In this case, we can note as well $y=h(x)=C x$.

Reformulating (1) in the new coordinates $x$, we can then arrive at

$$
\begin{aligned}
& \dot{x}=f(x)+\sum_{i=1}^{m} g_{i}(x) u_{i} \\
& y=h(x)
\end{aligned}
$$

where $x \in \mathcal{D} \subset \mathbb{R}^{n}$ with $n=2 n_{q}, u=\left[u_{1}, \cdots, u_{m}\right]^{T} \in$ $\mathbb{R}^{m}, y \in \mathbb{R}^{p}$ with $m \geq p$, and

$$
\begin{aligned}
& f(x)=\left[\begin{array}{c}
x_{2} \\
-M^{-1}\left(x_{1}\right) D\left(x_{1}, x_{2}\right) x_{2}-M^{-1}\left(x_{1}\right) K\left(x_{1}\right) x_{1}
\end{array}\right] \\
& {\left[g_{1}, \cdots, g_{m}\right]=\left[\begin{array}{c}
0 \\
M^{-1}\left(x_{1}\right) H^{T}\left(x_{1}\right)
\end{array}\right]}
\end{aligned}
$$

System (2) is typically nonlinear, and the concept of controllability has already been investigated in control community by applying differential geometric method [10]. The following will recall some basic notations of such a method.

For system (2), consider $f(x)=\left[f_{1}(x), \cdots, f_{n}(x)\right]^{T}$ as a vector field, i.e. $f(x)=\sum_{i=1}^{n} f_{i}(x) \frac{\partial}{\partial x_{i}}$ where $\frac{\partial}{\partial x_{i}}$ denotes the partial derivative in the direction of $e_{i}=$ $[0, \cdots, 0,1,0, \cdots, 0]^{T}$ whose $i$ th component is 1 . Then for any function $h_{i}(x)$, its Lie derivative in the direction of $f(x)$ is defined as

$$
L_{f(x)} h_{i}(x)=\frac{\partial h_{i}(x)}{\partial x} f(x)=\sum_{j=1}^{n} \frac{\partial h_{i}(x)}{\partial x_{j}} f_{j}(x)
$$

Iteratively, we can define the $j$ th Lie derivative as $L_{f}^{j} h_{i}=$ $L_{f} L_{f}^{j-1} h_{i}$ for $j \geq 1$.

For the configuration of soft robot described by the nonlinear system (2) with the chosen points $y=h(x) \in \mathbb{R}^{p}$, we can then define the relative degree for each $h_{i}(x)$ with $1 \leq i \leq p$.

Definition 1. [10] For system (2), the relative degree of $h_{i}(x)$ with $1 \leq i \leq p$ is noted as $r_{i}$ if the following conditions are satisfied for $x \in D$ :

$$
\left\{\begin{array}{l}
L_{g_{k}} L_{f}^{j-1} h_{i}=0, \quad \text { for all } 1 \leq k \leq m, 1 \leq j \leq r_{i}-1 \\
L_{g_{k}} L_{f}^{r_{i}-1} h_{i} \neq 0, \quad \exists k, \text { for } 1 \leq k \leq m
\end{array}\right.
$$

Then, system (2) is said to have the relative degree $r=$ $\sum_{i=1}^{p} r_{i}$.

Using the relative degree, we can introduce the concept of zero dynamics. Assume that system (2) has relative degree $r=\sum_{i=1}^{p} r_{i}$, then there exists a change of coordinates

$$
[z, \eta]^{T}=\left[h_{1}, \cdots, L_{f}^{r_{1}-1} h_{1}, \cdots, h_{p}, \cdots, L_{f}^{r_{p}-1} h_{p}, \eta\right]^{T}
$$

with $\eta \in \mathbb{R}^{n-r}$ being a complementary of $z$ to form a diffeomorphism (which is not unique), such that system (2) can be transformed into the following normal form:

$$
\begin{aligned}
& \dot{z}_{i}=A_{i} z_{i}+B_{i}\left[L_{f}^{r_{i}} h_{i}+\sum_{k=1}^{m} L_{g_{k}} L_{f}^{r_{i}-1} h_{i} u_{k}\right] \quad \forall i \in[1, p] \\
& \dot{\eta}=\alpha(z, \eta)+\beta(z, \eta) u
\end{aligned}
$$

where $z=\left[z_{1}^{T}, \cdots, z_{p}^{T}\right]^{T}, u=\left[u_{1}, \cdots, u_{m}\right]^{T}, \alpha(z, \eta)$ and $\beta(z, \eta)$ are determined by the chosen $\eta$, and

$A_{i}=\left[\begin{array}{ccccc}0 & 1 & 0 & \cdots & 0 \\ 0 & 0 & 1 & \cdots & 0 \\ \vdots & \vdots & \vdots & \ddots & \vdots \\ 0 & 0 & 0 & \cdots & 1 \\ 0 & 0 & 0 & \cdots & 0\end{array}\right] \in \mathbb{R}^{r_{i} \times r_{i}}, B_{i}=\left[\begin{array}{c}0 \\ 0 \\ \vdots \\ 0 \\ 1\end{array}\right] \in \mathbb{R}^{r_{i}}$ 
In control community, the sub-dynamics $\eta$ is named as zero dynamics of (3). Let us now define the decoupling matrix $\Gamma(x)$ as follows

$$
\Gamma(x)=\left[\begin{array}{ccc}
L_{g_{1}} L_{f}^{r_{1}-1} h_{1} & \cdots & L_{g_{m}} L_{f}^{r_{1}-1} h_{1} \\
\vdots & \ddots & \vdots \\
L_{g_{1}} L_{f}^{r_{p}-1} h_{p} & \cdots & L_{g_{m}} L_{f}^{r_{p}-1} h_{p}
\end{array}\right]
$$

based on which we can then state the following result.

Theorem 2. Under Assumption 1, the chosen points for the configuration of soft robots described by (1) are controllable if

$$
\operatorname{rank} \Gamma(x)=p, \forall x \in \mathcal{D}
$$

Proof: From (3), it is easy to obtain

$$
\mathcal{Y}=\Omega(x)+\Gamma(x) u
$$

with $\Gamma(x)$ being defined in (4) and

$$
\begin{aligned}
& \mathcal{Y}=\left[y_{1}^{\left(r_{1}\right)}, \cdots, y_{p}^{\left(r_{p}\right)}\right]^{T} \\
& \Omega(x)=\left[L_{f}^{r_{1}} h_{1}, \cdots, L_{f}^{r_{p}} h_{p}\right]^{T}
\end{aligned}
$$

Since $\Gamma(x) \in \mathbb{R}^{p \times m}$ with $p \leq m$, therefore, the condition (5) implies that $\Gamma(x) \Gamma^{T}(x) \in \mathbb{R}^{p \times p}$ and

$$
\operatorname{rank} \Gamma(x) \Gamma^{T}(x)=p, \forall x \in \mathcal{D}
$$

Setting

$$
u=\Gamma^{T}(x)\left[\Gamma(x) \Gamma^{T}(x)\right]^{-1}[-\Omega(x)+v]
$$

where $v=\left[v_{1}, \cdots, v_{p}\right]^{T}$ is a virtual control input which can be freely chosen by the designer, then the input-output relation (6) can be linearized as

$$
y_{i}^{\left(r_{i}\right)}=v_{i}, \forall i \in[1, p]
$$

from which we can draw the conclusion that the chosen points $y \in \mathbb{R}^{p}$ are controllable.

It is worth noting that the rank condition (5) gives only a sufficient condition to check whether the chosen points of interest are controllable or not. Also, the verification of the rank condition (5) depends on the computation of Lie derivative of $h_{i}$ in the direction of $f$ and $g_{i}$, which are normally high dimensional. It is therefore interesting (or even necessary) to investigate the approach to deduce the order of system (2).

\section{ANALYSIS BASED ON REDUCED-ORDER MODEL}

The key idea of model-order reduction is to seek a lowdimensional state space where the dynamics of the highdimensional system can be almost kept, by neglecting the states which are hard to be reached and hard to be observed. The requirements when applying model order reduction are numerous [21]. First, the approximation error should be small (in the sense of input-output norm, or least square). Also, the interested properties, such as stability and passivity, should be preserved. Especially, the procedure needs to be computationally efficient, stable and automatic.
For linear system, the well-known balanced-truncation method can be applied to minimize $H_{\infty}$ and Hankel norms [21], which however is not any more valid for nonlinear system described as (2). Here, we adopt the technique based on Karhunen-Loève transform [22] (named as well POD: proper orthogonal decomposition [16]). This method enables us to seek the best approximating low-dimensional subspace (by solving a $\mathrm{H}_{2}$ optimization problem, which needs only linear matrix computation even for nonlinear systems), and then a Galerkin projection can be applied to obtain lowdimensional nonlinear systems [23], [20].

In the proposed methodology, the POD method is adopted (other MOR methods can also be used). This method is based on empirical data, which can be generated from numerical simulation of high-dimensional nonlinear dynamical system (2). The details of this technique can be found in any textbook on model-order reduction, and the following will only briefly present the idea.

For the system (2) obtained from FEM model, denote by $\mathcal{U}_{S}=\left\{u_{s_{1}}, \cdots, u_{s_{N}}\right\}$ the sequence of control inputs, and $\mathcal{X}_{S}=\left\{x_{s_{1}}, \cdots, x_{s_{N}}\right\}$ as the corresponding samples of $x(t)$. Note $\mathcal{S} \subset \mathbb{R}^{n}$ as the subspace and $\Pi$ as the projection operator mapping $\mathbb{R}^{n}$ onto $\mathcal{S}$, i.e.,

$$
\Pi: \mathbb{R}^{n} \rightarrow \mathcal{S}
$$

The objective of POD is to search such an operator for the purpose of minimizing the following cost function:

$$
\mathcal{J}(\Pi)=\sum_{i=1}^{N}\left\|x_{s_{i}}-\Pi x_{s_{i}}\right\|_{2}^{2}
$$

For this, define the correlation matrix of the empirical data

$$
\mathcal{X}_{\text {cov }}=\sum_{i=1}^{N} x_{s_{i}} x_{s_{i}}^{T}
$$

and it has been proven [21] that the optimal $k$-dimensional subspace satisfies:

$$
\min _{\Pi} \mathcal{J}(\Pi)=\sum_{i=n-k+1}^{n} \sigma_{i}
$$

where $\sigma_{i}$ represents the eigenvalues of $\mathcal{X}_{\text {cov }}$ with decreasing order as $\sigma_{1} \geq \sigma_{2} \geq \cdots \geq \sigma_{n}$. This implies that the solution of the above optimization problem is equivalent to the singular value decomposition of $\mathcal{X}$ (noted as $\mathcal{X}=$ $U \Sigma V^{T}$ ), and the optimal projector is the first $k$ rows of the left singular vector of $\mathcal{X}$ (i.e. $\Pi=\operatorname{col}\left[U_{1}, \cdots, U_{k}\right] \in \mathbb{R}^{k \times n}$ ) with the property $\Pi \Pi^{T}=I$.

With the deduced $k$-dimensional projector $\Pi$, the trajectory $x(t)$ can be then projected onto the subspace $\mathcal{S}$ as

$$
\xi=\Pi x
$$

where $\xi \in \mathbb{R}^{k}$ is the new coordinates on $\mathcal{S}$. This projection enables us to apply Galerkin method to deduce the reducedorder model. Precisely, substituting (7) back into the highdimensional system (2) yields

$$
\begin{aligned}
& \dot{\xi}=\bar{f}(\xi)+\sum_{i=1}^{m} \bar{g}_{i}(\xi) u_{i} \\
& y=\bar{h}(\xi)
\end{aligned}
$$


with

$$
\begin{aligned}
& \bar{f}(\xi)=\Pi f\left(\Pi^{T} \xi\right) \\
& \bar{g}_{i}(\xi)=\Pi g_{i}\left(\Pi^{T} \xi\right) \\
& \bar{h}(\xi)=h\left(\Pi^{T} \xi\right)
\end{aligned}
$$

Note that system (8), which is of low-dimensional $k$, has the same structure as the high-dimensional system (2). Similarly, we can calculate the relative degree for (8), noted as $\left(\bar{r}_{1}, \cdots, \bar{r}_{p}\right)$, and then define the decoupling matrix $\bar{\Gamma}(\xi)$ for (8) as follows

$$
\bar{\Gamma}(\xi)=\left[\begin{array}{ccc}
L_{\bar{g}_{1}} L_{\bar{f}}^{\bar{r}_{1}-1} \bar{h}_{1} & \cdots & L_{\bar{g}_{m}} L_{\bar{f}}^{\bar{r}_{1}-1} \bar{h}_{1} \\
\vdots & \ddots & \vdots \\
L_{\bar{g}_{1}} L_{\bar{f}}^{\dot{\bar{r}}_{p}-1} \bar{h}_{p} & \cdots & L_{\bar{g}_{m}} L_{\overline{\bar{f}}}^{\bar{r}_{p}-1} \bar{h}_{p}
\end{array}\right]
$$

based on which we can state similar result as that of Theorem 2 , i.e., the chosen points of interest for the configuration of soft robots described by (1) are controllable if $\operatorname{rank} \bar{\Gamma}(\xi)=p$.

\section{SOFT ROBOT PRE-CHECKING PROCEDURE}

Having an idea to design a soft robot under Assumption 1 , the proposed rank conditions enable us to pre-check numerically the controllability of the points of interest in a virtual environment before the fabrication of prototype. Due to the deduced rank conditions, an iterative pre-checking procedure can be established, which is illustrated in Figure. 2.

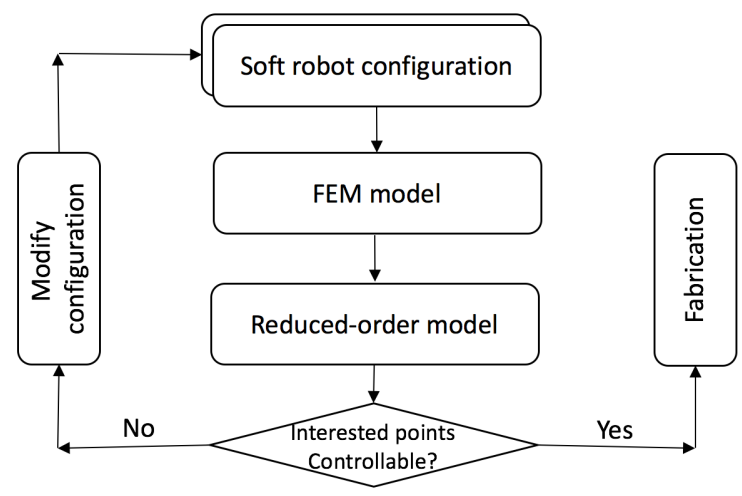

Fig. 2. Iterative procedure for the design of controllable soft robotics

Precisely, the users draw a configuration of soft robot they want to design in a virtual environment via existing software, like CAD. Under Assumption 1, a FEM model might be then deduced automatically by using certain software (such as SOFA [24]), once the flexible material properties (Young's modulus, deformation law...) have been determined. After that, the users can choose certain points of interest to check whether those points are controllable. To realize this, a low-dimensional model can be obtained, by applying MOR techniques, such as POD presented in Section IV. If the rank condition presented in Section IV is not satisfied, then we need to modify the configuration of soft robot ( change either the structure of robot, or the placement and the type of actuators), and repeat the numerical design procedure. Once a feasible configuration is found out, i.e., the rank condition is satisfied, the users can then pass to the stage of fabrication.
By using the proposed methodology, the users can largely reduce the design duration. Also, the designed robot has been numerically verified in the virtual environment, which guarantees the feasible functionalities of the final prototype, provided that all conditions in Assumption 1 are fulfilled as well during the fabrication.

\section{CASE STUDY}

In this section, we are going to use the proposed methodology to design a parallel soft robot and pre-check the controllability of the points of interest before its real fabrication. Using CAD kind of software, we can draw the possible configuration of such a parallel robot, for example the one presented in [5]. Figure 3 depicts one configuration with 4 soft links actuated by 4 independent cables.

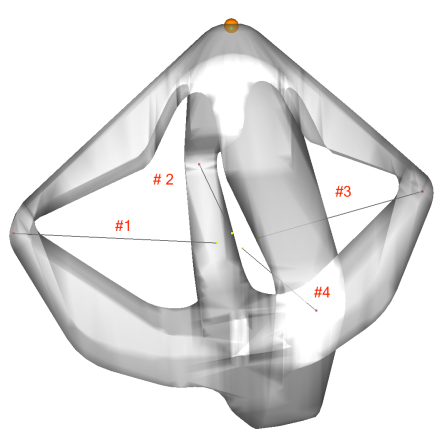

Fig. 3. Possible configuration of the parallel soft robot, actuated by 4 fixed cables; red point on the top represents the point of interest.

In order to specify the flexible characteristic, a constitutive law needs to be chosen. Since the robot will be made of silicone, therefore we use a co-rotational elastic formulation. This formulation assumes a linear elastic deformation law for the elements (tetrahedra in this case), but accounts for large rotations by formulating that pure elastic deformation within the frame of each element. This offers a reasonable model, which can account for large rotations of the elements. More details about the co-rotational formulation can be found in [25]. The model is parameterized by Young's Modulus and Poisson's ratio. To deduce the FEM model, a mesh of 1628 nodes and 4147 tetrahedra is defined, and the value of Young's Modulus is set to 500Mpa and the Poisson's ration is set to 0.45 (quasi incompressible). With 3 degrees of freedom (Dof) per node, the number of degrees of freedom of that model is $\operatorname{dim} x=3 \times 1628=4884$, which is displayed in Figure 4.

To reduce the high number of Dof, we proceed to apply a projection-based model-order reduction method, as described previously. In practice, the robot is simulated in all its possible deformations, by applying all possible actuations to make the robot explore its entire workspace. All the data is stored in a large matrix called the snapshot matrix. The reduced basis is computed by applying POD to the stored states, i.e., the snapshot matrix. Using an error tolerance of $10^{-3}$, a reduced basis of 50 vectors is selected, i.e., $\operatorname{dim} \xi=50$ in (8). 


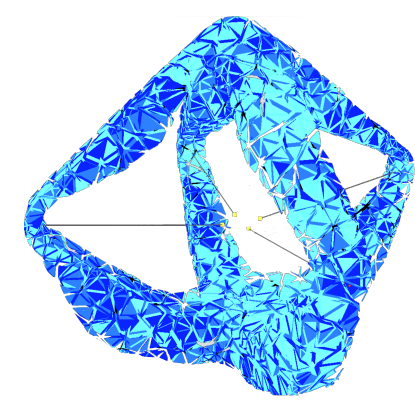

Fig. 4. Finite-element model of the soft parallel robot for the configuration described in Figure 3.

In this case study, we want to check whether the top point of this parallel robot is controllable or not. Therefore, it has been selected as the output of system (2). Several scenarios have been checked by calculating the rank of decoupling matrix $\bar{\Gamma}(\xi)$ defined in (9). The results are listed as follows:

\begin{tabular}{|c|c|c|c|}
\hline Scenario & Actuated cables & $\operatorname{rank} \Gamma(\xi)$ & Conclusion \\
\hline 1 & $\# 1, \# 2$ & 2 & 2D contr. \\
2 & $\# 1, \# 3$ & 2 & 2D contr. \\
3 & $\# 1, \# 2, \# 3, \# 4$ & 3 & 3D contr. \\
\hline
\end{tabular}

The results listed in the above table show that, using only 2 cables (either cable $\# 1$ and $\# 2$, or cable $\# 1$ and $\# 3$ ), the top point can be controlled only in 2 dimensions, while using all cables enables us to control the top point in 3 dimensional space. The proposed methodology is validated by this parallel soft robot since the conditions drawn from the rank condition coincide well the reality.

\section{CONCLUSION}

This paper proposed a method on how to design a controllable silicone soft robot based on FEM: from concept to prototype. Firstly, we applied FEM to obtain a highdimensional nonlinear dynamical system. Then, the controllability of certain points of interest has been checked by using differential geometric method, which is a quite popular approach in control community. The deduced rank condition is based on the high-dimensional system, thus is computationally expensive. Consequently, a reduced-order system was deduced by applying MOR and Galerkin projection, for which the rank condition was re-formulated. An iterative design procedure has been proposed to facilitate the design of controllable soft robots, and its efficiency has been highlighted by designing a parallel soft robot.

\section{REFERENCES}

[1] D. Trivedi, C. D. Rahn, W. M. Kier, and I. D. Walker, "Soft robotics: Biological inspiration, state of the art, and future research," Appl. Bionics Biomechanics, vol. 5, no. 3, pp. 99-117, 2008.

[2] S. Kim, C. Laschi, and B. Trimmer, "Soft robotics: a bioinspired evolution in robotics," Trends in Biotechnology, vol. 31, no. 5, pp. $287-294,2013$.

[3] F. Renda, M. Giorelli, M. Calisti, M. Cianchetti, and C. Laschi, "Dynamic model of a multibending soft robot arm driven by cables," IEEE Transactions on Robotics, vol. 30, no. 5, pp. 1109-1122, 2014.
[4] N. Koenig and A. Howard, "Design and use paradigms for gazebo, an open-source multi-robot simulator," in 2004 IEEE/RSJ International Conference on Intelligent Robots and Systems (IROS) (IEEE Cat. No.04CH37566), vol. 3, Sept 2004, pp. 2149-2154 vol.3.

[5] C. Duriez, "Control of elastic soft robots based on real-time finite element method," in Robotics and Automation (ICRA), 2013 IEEE International Conference on. IEEE, 2013, pp. 3982-3987.

[6] C. Duriez and T. Bieze, "Soft robot modeling, simulation and control in real-time," Soft Robotics: Trends, Applications and Challenges: Proceedings of the Soft Robotics Week, April 25-30, 2016, Livorno, Italy, vol. 17, p. 103, 2016.

[7] M. Thieffry, A. Kruszewski, O. Goury, T.-M. Guerra, and C. Duriez, "Dynamic control of soft robots," in IFAC World Congress, 2017.

[8] E. Coevoet, T. Morales-Bieze, F. Largilliere, Z. Zhang, M. Thieffry, M. Sanz-Lopez, B. Carrez, D. Marchal, O. Goury, J. Dequidt, and C. Duriez, "Software toolkit for modeling, simulation and control of soft robots," Advanced Robotics, pp. 1-26, 2017.

[9] F. Largilliere, V. Verona, E. Coevoet, M. Sanz-Lopez, J. Dequidt, and C. Duriez, "Real-time Control of Soft-Robots using Asynchronous Finite Element Modeling," in ICRA 2015, SEATTLE, United States, May 2015, p. 6. [Online]. Available: https://hal.inria.fr/hal-01163760

[10] A. Isidori, "Nonlinear control systems (3rd edition)," London: Springer-Verlag, 1995.

[11] G. Zheng, D. Boutat, and J. Barbot, "Output dependent observability linear normal form," in Decision and Control, 2005 and 2005 European Control Conference. CDC-ECC'05. 44th IEEE Conference on. IEEE, 2005, pp. 7026-7030.

[12] K. Zhou, J. C. Doyle, K. Glover, et al., Robust and optimal control. Prentice hall New Jersey, 1996, vol. 40.

[13] B. Beliczynski, I. Kale, and G. D. Cain, "Approximation of fir by iir digital filters: An algorithm based on balanced model reduction," IEEE Transactions on Signal Processing, vol. 40, no. 3, pp. 532-542, 1992.

[14] C. W. Rowley, "Model reduction for fluids, using balanced proper orthogonal decomposition," in Modeling And Computations In Dynamical Systems: In Commemoration of the 100th Anniversary of the Birth of John von Neumann. World Scientific, 2006, pp. 301-317.

[15] A. K. Noor and J. M. Peters, "Reduced basis technique for nonlinear analysis of structures," Aiaa journal, vol. 18, no. 4, pp. 455-462, 1980.

[16] C. W. Rowley, T. Colonius, and R. M. Murray, "Model reduction for compressible flows using pod and galerkin projection," Physica D: Nonlinear Phenomena, vol. 189, no. 1-2, pp. 115-129, 2004.

[17] S. Gugercin and A. C. Antoulas, "A survey of model reduction by balanced truncation and some new results," International Journal of Control, vol. 77, no. 8, pp. 748-766, 2004.

[18] Z. Bai, "Krylov subspace techniques for reduced-order modeling of large-scale dynamical systems," Applied Numerical Mathematics, vol. 43, no. 1, pp. 9 - 44, 2002, 19th Dundee Biennial Conference on Numerical Analysis. [Online]. Available: http://www.sciencedirect.com/science/article/pii/S0168927402001162

[19] F. Chinesta, P. Ladeveze, and E. Cueto, "A short review on model order reduction based on proper generalized decomposition," Archives of Computational Methods in Engineering, vol. 18, no. 4, p. 395, 2011.

[20] O. Goury and C. Duriez, "Fast, generic and reliable control and simulation of soft-robots using model order reduction," IEEE Transactions on Robotics, submitted, 2017.

[21] A. C. Antoulas, Approximation of Large-Scale Dynamical Systems (Advances in Design and Control) (Advances in Design and Control). Philadelphia, PA, USA: Society for Industrial and Applied Mathematics, 2005.

[22] A. J. Newman, "Model reduction via the karhunen-loeve expansion part i: An exposition," Tech. Rep., 1996.

[23] O. Goury and C. Duriez, "Projection-based model order reduction for real-time control of soft robots," in XIV International Conference on Plasticity. Fundamentals and Applications, 2017.

[24] J. Allard, S. Cotin, F. Faure, P.-J. Bensoussan, F. Poyer, C. Duriez, H. Delingette, and L. Grisoni, "Sofa-an open source framework for medical simulation," in MMVR 15-Medicine Meets Virtual Reality, vol. 125. IOP Press, 2007, pp. 13-18.

[25] M. Nesme, Y. Payan, and F. Faure, "Efficient, Physically Plausible Finite Elements," in Eurographics, 2005. 